\title{
Downlink transmission and channel estimation for cell-free massive MIMO-OFDM with DSDs
}

\author{
Yunxiang Guo ${ }^{1}$, Zhenqi Fan², An Lu², Pan Wang ${ }^{3}$, Dongjie Liư ${ }^{3}$, Xinjiang Xia ${ }^{3^{*}}$ and Dongming Wang $1,3^{*}$
}

\author{
*Correspondence: \\ xiaxinjiang@pmlabs.com.cn; \\ wangdm@seu.edu.cn \\ ${ }^{1}$ National Mobile \\ Communications Research \\ Laboratory, Southeast \\ University, Nanjing 210096, \\ People's Republic of China \\ ${ }^{3}$ Ubiquitous Communication \\ Research Center, Purple \\ Mountain Laboratories, \\ Nanjing 211111, People's \\ Republic of China \\ Full list of author information \\ is available at the end of the \\ article
}

\begin{abstract}
In a cell-free massive MIMO system, multiple users arrive at multiple access points at separate times, while in an OFDM system, different delays can be equivalent to delay spread differences (DSDs). Since DSDs are not all the same, in the downlink transmission process, it is necessary to consider its impact on transmission techniques, such as channel estimation and downlink precoding. In this paper, aiming at the performance loss caused by DSDs in cell-free massive MIMO-OFDM system, we propose a multi-RB precoding optimization algorithm that maximizes the downlink sum rate. We derive the sum rate maximization problem into an iterative second-order cone programming form to achieve convex approximation. Then, considering the impact of DSDs on the accuracy of cell-free massive MIMO-OFDM channel estimation, we propose a downlink channel estimation method, which jointly uses channel state information reference signal and demodulation reference signal. The simulation results show that the proposed multi-RB optimal precoding can effectively improve the downlink sum rate, and the proposed downlink channel estimation can obtain accurate multi-RB frequencydomain channel parameters.
\end{abstract}

Keywords: Cell-free massive MIMO-OFDM, Delay spread differences, Downlink channel estimation, Second-order cone program, Convex approximation

\section{Introduction}

A cell-free system is a new concept of mobile communication system architecture derived from distributed MIMO, in which the antenna unit is separated from the processing unit. Multiple remote antenna units (RAUs) are connected to the central processing unit (CPU) through optical fibers, and joint signal processing is performed through the CPU. Compared with the cellular system that eliminates the interference between antennas by allocating different frequencies, the joint signal processing of the cell-free system can eliminate the interference between antennas on the same frequency by means of simultaneous equations that make it possible to improve spectrum efficiency, coverage capability and cell boundary performance [1-4].

However, due to the distributed characteristics of cell-free systems, similar to coordinated multipoint (CoMP) systems or 5G-based IoT, different user equipment (UE) and author(s) and the source, provide a link to the Creative Commons licence, and indicate if changes were made. The images or other third party material in this article are included in the article's Creative Commons licence, unless indicated otherwise in a credit line to the material. If material is not included in the article's Creative Commons licence and your intended use is not permitted by statutory regulation or exceeds the permitted use, you will need to obtain permission directly from the copyright holder. To view a copy of this licence, visit http:// creativecommons.org/licenses/by/4.0/. 
RAUs have separate propagation delays. These variations will cause the receiver to synchronize with the transmitted signal that arrives first, and DSDs will be generated on the asynchronous transmitted signal that arrives later. Especially in the OFDM system, accurate symbol time synchronization is an important prerequisite for removing the cyclic prefix $(\mathrm{CP})$ and completing correct demodulation. DSDs will cause the received signal to produce a phase rotation that varies with the subcarrier index during the demodulation process, which will have a negative impact on system performance [5-11].

The major contributions of this paper are summarized as follows.

1. A multi-RB precoding optimization algorithm to maximize the cell-free massive MIMO system sum rate under the total RAU power constraint is proposed.

2. A DMRS channel estimation is proposed.

The novelty of this paper is to solve the problem that the performance loss of DSDs to broadband precoding increases as the number of subcarriers increases since per subcarrier precoding is not supported in the current 5 G NR protocol [12].

The following notations are used. All boldface letters represent vectors (lower case) or matrices (upper case). The $P \times P$ identity matrix is denoted $\mathbf{I}_{P}$. The notations $\mathbb{C}^{N}$ and $\mathbb{C}^{M \times N}$ refer to complex $N$-dimensional vectors and $M \times N$ matrices, respectively. The operators $\operatorname{diag}(\cdot),(\cdot)^{T}$ and $(\cdot)^{H}$ denote diagonalization, transpose and conjugate transpose, respectively. A random vector $x \sim \mathcal{C N}(\mathbf{m}, \boldsymbol{\Phi})$ is a complex Gaussian distribution with a mean vector $\mathbf{m}$ and covariance matrix $\boldsymbol{\Phi}$.

\section{Problem formulation}

\subsection{Impact of the DSDs}

In an OFDM system, obtaining accurate sampling of the transmitted signal within the symbol period is the prerequisite for the receiver to perform FFT. For this reason, it is necessary to perform symbol timing synchronization after removing CP. However, in the cell-free system as shown in Fig. 1, because the distances from RAUs to UEs are different, the receiver performs symbol timing synchronization with the signal that arrives first, and the signal that arrives subsequently will produce $\tau$ samples DSDs during the sampling process, as shown in Fig. 2. After FFT, $\tau$ samples DSDs result in a phase rotation of $e^{-j 2 \pi n \tau / N_{c}}$ on the $n$th subcarrier, where $N_{c}$ is the number of FFT points. It can be seen that phase rotation increases with the increase of subcarrier index, and the impact of phase rotation will be superimposed in the coherence bandwidth, causing additional frequency selective fading of the channel.

\subsection{System model}

We consider a cell-free massive MIMO-OFDM system, including $M$ multi-antenna RAUs and $K$ single-antenna UEs, where each RAU has $L$ antennas. The locations of RAUs and UEs are randomly distributed within the coverage area. The total number of subcarriers in the system is $N$, UEs are grouped into $N_{\mathrm{RB}}$ resource blocks (RBs), and each $\mathrm{RB}$ contains $\lambda_{\mathrm{RB}}=N / N_{\mathrm{RB}}$ subcarriers. The number of UEs in each RB is much smaller than the total number of RAUs. 

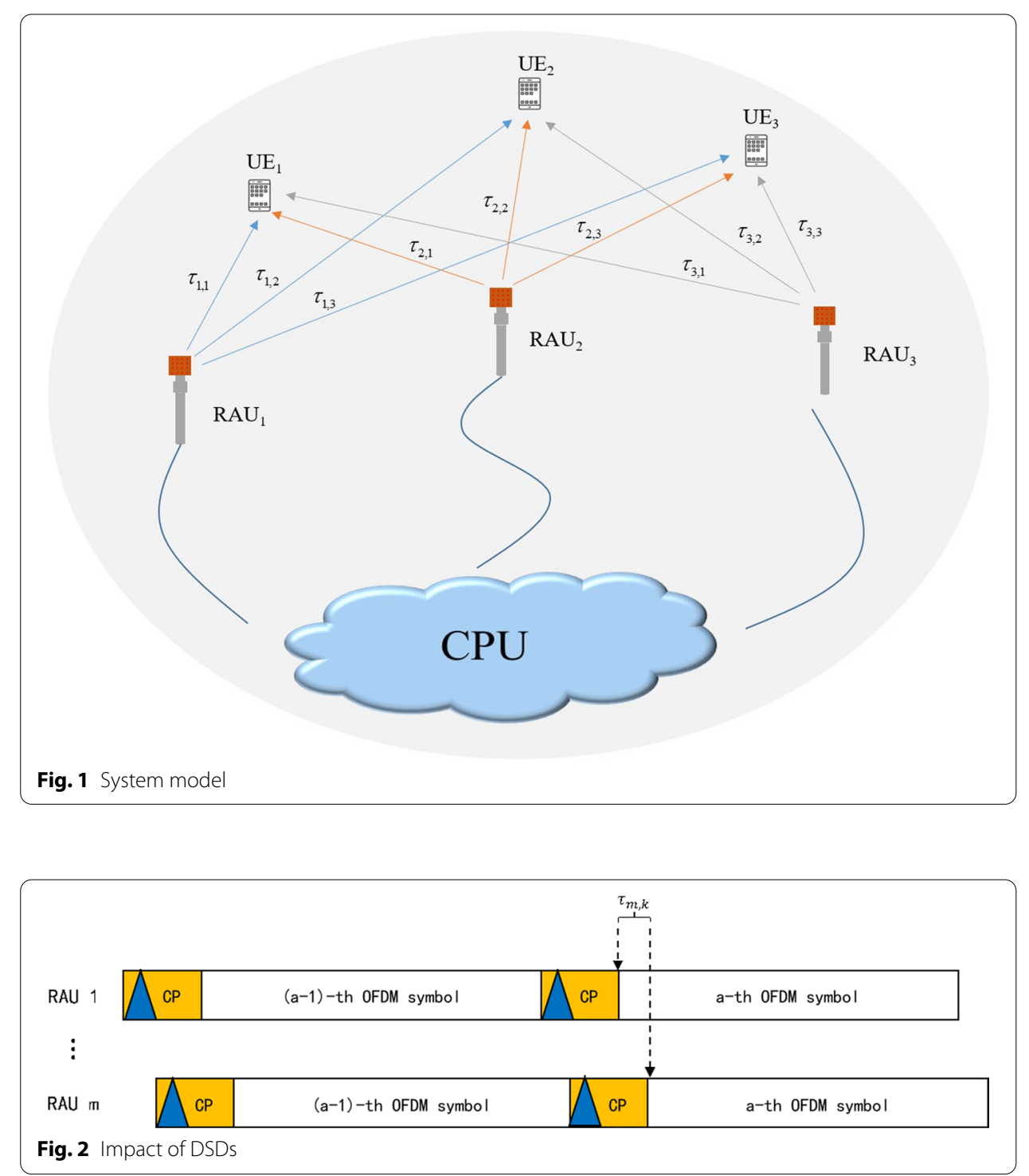

The frequency-domain symbol sequence sent by RAU $m$ on the th OFDM symbol is $\mathbf{x}_{m}=\left[x_{m, 0}, \ldots, x_{m, n}, \ldots, x_{m, N-1}\right]^{T}$. The time-domain symbol sequence obtained after the $N_{c}$-point IFFT at the transmitter is $\mathbf{x}_{m}[t]=\left[x_{m, 0}[t], \ldots, x_{m, n^{\prime}}[t], \ldots, x_{m, N-1}[t]\right]^{T}$, where $x_{m, n^{\prime}}[t]=\frac{1}{N} \sum_{n=0}^{N-1} x_{m, n} e^{j 2 \pi n n^{\prime} / N_{c}}$. According to the derivation in [6], when the signal arrives at the receiver, due to the DSDs of $\tau$ samples, $x_{m, n^{\prime}}[t] \rightarrow x_{m, n^{\prime}}[t-\tau]$, after $N_{c}$-point FFT, a phase rotation $\Theta=e^{-j 2 \pi n \tau / N_{c}}$ is generated on the frequency-domain symbol of the $n$th subcarrier. Under the impact of DSDs, the channel vector $\mathbf{h}_{m, k, n} \in C^{L \times 1}$ of the $m$ th RAU and the $k$ th UE on the $n$th subcarrier can be expressed as

$$
\mathbf{h}_{m, k, n}=\beta_{m, k}^{1 / 2} \mathbf{g}_{m, k, n} \Theta_{m, k, n}
$$

where $\beta_{m, k}=\left(d_{m, k}\right)^{-l}$ is the large-scale fading coefficient independent of frequency, $d_{m, k}$ is the distance between the $m$ th RAU and the $k$ th UE, and $l$ is the path loss exponent. $\Theta_{m, k, n}=e^{-j 2 \pi n \tau_{m, k} / N_{c}}$ is the phase rotation caused by DSDs, where 
$\tau_{m, k}=d_{m, k} / c-\tau_{k}^{s y n}$ is the DSDs between the $m$ th RAU and the $k$ th UE, where $\tau_{k}^{s y n}=\min _{m} \tau_{m, k}$ is the reference synchronization time and $c$ is the speed of light.

Considering the downlink transmission, we model the received signal of UE $k$ on the $n$th subcarrier as

$$
y_{k, n}=\mathbf{h}_{k, n}^{H} \mathbf{w}_{k} s_{k, n}+\sum_{u \neq k}^{K} \mathbf{h}_{k, n}^{H} \mathbf{w}_{u} s_{u, n}+z_{k, n}
$$

where

$$
\mathbf{h}_{k, n}=\left[\mathbf{h}_{1, k, n}, \ldots, \mathbf{h}_{M, k, n}\right]^{T} \in \mathbb{C}^{M L \times 1}
$$

represents the frequency-domain channel vector on the $n$th subcarrier from all RAUs to downlink UE $k, s_{k, n} \sim \mathcal{C N}(0,1)$ is the data symbol sent by all RAUs to downlink UE $k$, $z_{k, n} \sim \mathcal{C N}\left(0, \sigma_{k, n}^{2}\right)$ is additive white Gaussian noise,

$$
\mathbf{w}_{k}=\left[\mathbf{w}_{1, k}, \ldots, \mathbf{w}_{M, k}\right]^{T} \in \mathbb{C}^{M L \times 1}
$$

represents the precoding vector on the $n$th subcarrier for the data stream of downlink UE $k$, where $\mathbf{w}_{m, k} \in \mathbb{C}^{L \times 1}$. Assume that the precoding granularity is $\varphi$, the same precoding vector is used on the adjacent $N_{\varphi}=\varphi \lambda_{\mathrm{RB}}$ subcarriers, and there are a total of $R=N / N_{\varphi}$ different precoding vectors in the system bandwidth. That is, for any $n=(r-1) N_{\varphi}+1, \ldots, r N_{\varphi}$, where $r=1, \ldots, R$, we have $\mathbf{w}_{k, n}=\mathbf{w}_{k}$. Therefore, the received signal of downlink UE $k$ within the system bandwidth can be modeled as

$$
y_{k}=\sum_{n=1}^{N} \mathbf{h}_{k, n}^{H} \mathbf{w}_{k} s_{k, n}+\sum_{u \neq k}^{K} \sum_{n=1}^{N} \mathbf{h}_{k, n}^{H} \mathbf{w}_{u} s_{u, n}+z_{k, n}
$$

\section{Proposed approach}

\subsection{Proposed precoding design}

In this paper, we are interested in the problem of the sum rate maximization under total RAU power constraints. On the $n$th subcarrier, the SINR of downlink UE $k$ is

$$
\gamma_{k, n}=\frac{\left|\mathbf{h}_{k, n}^{H} \mathbf{w}_{k}\right|^{2}}{\sum_{u \neq k}^{K}\left|\mathbf{h}_{k, n}^{H} \mathbf{w}_{u}\right|^{2}+\sigma_{k}^{2}}
$$

Then, the design problem is given by

$$
\begin{aligned}
& \max _{\mathbf{w}_{k}} \sum_{n=1}^{N} \sum_{k=1}^{K} \log _{2}\left(1+\gamma_{k . n}\right) \\
& \text { s.t. } \sum_{k=1}^{K}\left\|\mathbf{w}_{k}\right\|^{2} \leqslant P_{\max }, \forall k
\end{aligned}
$$


Since problem (7) is NP-hard, the globally optimal design mainly acts as a theoretical benchmark rather than a practical solution. Herein, motivated by [13, 14], we develop a low-complexity algorithm that satisfies the necessary optimal conditions of (7).

According to the monotonicity of the logarithmic function, (7) is equivalent to

$$
\begin{aligned}
& \max _{\mathbf{w}_{k}} \prod_{n=1}^{N} \prod_{k=1}^{K}\left(1+\gamma_{k, n}\right) \\
& \text { s.t. } \sum_{k=1}^{K}\left\|\mathbf{w}_{k}\right\|^{2} \leqslant P_{\max }, \forall k
\end{aligned}
$$

which can be equivalently rewritten as

$$
\begin{aligned}
& \max _{\mathbf{w}_{k}, \mathbf{t}_{k, n}} \prod_{n=1}^{N} \prod_{k=1}^{K} f_{k, n} \\
& \text { s.t. } \sum_{k=1}^{K}\left\|\mathbf{w}_{k}\right\|^{2} \leqslant P_{\max }, \forall k \\
& \gamma_{k, n} \geqslant f_{k, n}-1, \forall k \\
& f_{k, n} \geqslant 1, \forall k
\end{aligned}
$$

Note that the objective function in (9) admits an SOC representation. Since (9b) and (9d) are already convex forms, we mainly focus on dealing with the constraints in (9c).

First, (9c) can be rewritten as

$$
\sum_{u \neq k}^{K}\left|\mathbf{h}_{k, n}^{H} \mathbf{w}_{u}\right|^{2}+\sigma_{k}^{2} \leqslant \frac{\mathbf{w}_{k}^{H} \boldsymbol{\Lambda}_{k, n} \mathbf{w}_{k}}{f_{k, n}-1}
$$

where $\boldsymbol{\Lambda}_{k, n}=\mathbf{h}_{k, n} \mathbf{h}_{k, n}^{H}$. We can see that (10) is also non-convex, since the right side of (10) has the form of quadratic-over-linear, it can be replaced by its first-order expansions [15]. Thus, we define

$$
J(\mathbf{w}, f, \Lambda)=\frac{\mathbf{w}^{H} \boldsymbol{\Lambda} \mathbf{w}}{f-\alpha}
$$

where $\boldsymbol{\Lambda} \geq 0$ and $f \geq \alpha$. We obtain the first-order Taylor expansion of (11) about a certain point $\left(\mathbf{w}^{(a)}, f^{(a)}\right)$ as

$$
\begin{aligned}
& J\left(\mathbf{w}, f, \mathbf{w}^{(a)}, f^{(a)}, \boldsymbol{\Lambda}, \alpha\right) \\
& \quad=\frac{2 \Re\left\{\left(\mathbf{w}^{(a)}\right)^{H} \boldsymbol{\Lambda} \mathbf{w}\right\}\left(f^{(a)}-\alpha\right)-\left(\mathbf{w}^{(a)}\right)^{H} \boldsymbol{\Lambda} \mathbf{w}^{(a)}(f-\alpha)}{\left(f^{(a)}-\alpha\right)^{2}}
\end{aligned}
$$

From the above analysis, we can transform the constraint of (9c) into a convex form 


$$
\sum_{u \neq k}^{K}\left|\mathbf{h}_{k, n}^{H} \mathbf{w}_{u}\right|^{2}+\sigma_{k}^{2} \leqslant J\left(\mathbf{w}_{k}, f_{k}, \mathbf{w}_{k}^{(a)}, f_{k}^{(a)}, \boldsymbol{\Lambda}_{k}, 1\right)
$$

Finally, the original problem (8) can be reformulated as a convex approximate problem (14) which can be solved in Algorithm 1.

$$
\begin{aligned}
& \max _{\left\{\mathbf{w}_{k}, \mathbf{t}_{k, n}\right\}} \prod_{n=1}^{N} \prod_{k=1}^{K} f_{k, n} \\
& \text { s.t. }(9 b),(9 d),(13)
\end{aligned}
$$

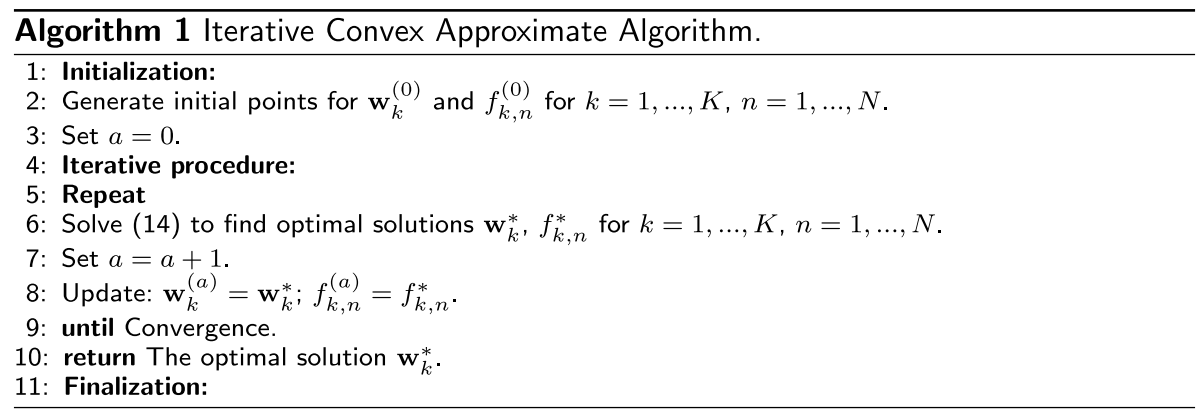

\subsection{Downlink channel estimation}

In this section, considering the impact of DSDs on the accuracy of cell-free massive MIMO-OFDM system channel estimation, we propose a high-precision multi-RB downlink channel estimation scheme, which provides the necessary channel information for multi-RB precoding optimization. In the pilot design of the 5G NR [16], CSI-RS is mainly used for channel sounding to obtain the path loss and DSD between UEs and RAUs. DMRS is mainly used for the demodulation of uplink and downlink data. The least squares (LS) algorithm is usually used to estimate the initial channel response of the pilot position, and then, interpolation filtering is performed in the frequency domain to obtain the channel response of the data position [17].

Regarding the CSI-RS of cell-free system, in uplink, sounding reference signal (SRS) is used to collect DSDs and large-scale fading; in downlink, tracking reference signal (TRS), a special CSI-RS, is used to track the phase deviation on the received signal.

For SRS, per UE tracking can be achieved in the 5G NR protocol. Therefore, the uplink channel estimation can be implemented in CPU. For downlink channel estimation, the signal overhead for per AP tracking is too large, and the implementation complexity is too high for UEs. Therefore, to estimate the statistical properties of downlink channels, we choose to use a composite channel tracking method, which is discussed in the following specific steps.

The specific steps of the multi-RB channel estimation we proposed are as follows:

First, all RAUs transmit orthogonal CSI-RS, UE $k$ estimates large-scale fading, $\beta_{1}^{1 / 2}, \beta_{2}^{1 / 2}, \ldots, \beta_{M}^{1 / 2}$ and DSDs, $\tau_{1}, \tau_{2}, \ldots, \tau_{M}$ of the overall channel with all RAUs based on the reference signal. It is worth mentioning that all RAUs can also send the same CSI-RS 
pilot, that is, the CSI-RS pilot adopts a single-port design. At this time, the receiver only estimates the composite signal from all RAUs to the UE. Specifically, multiple RAUs are regarded as multipath signals, and the power and delay of each path are estimated on UE $k$ based on the distinguishable time-domain multipath signals. The single-port design can effectively reduce the pilot overhead and design complexity, but it will produce additional channel time/frequency selectivity. In the case of sufficient estimation accuracy, these two methods are theoretically equivalent.

Second, considering that there are $P$ equally spaced DMRS in the coherent bandwidth, after LS, the frequency-domain signal collected by UE $k$ is

$$
\mathbf{y}_{N_{P}}=\left[y_{N_{1}}, y_{N_{2}}, \ldots, y_{N_{P}}\right]^{T} \in \mathbb{C}^{P \times 1}
$$

For convenience of representation, the subscript $k$ is omitted in the following notation. On subcarrier $p$,

$$
y_{N_{p}}=\sum_{m=1}^{M} \beta_{m}^{1 / 2} \Theta_{m, N_{p}} \mathbf{g}_{m, N_{p}}^{T} \mathbf{w}_{m}+z_{N_{p}}
$$

The precoding vector of the channel between UE $k$ and RAU $m$ is $\mathbf{w}_{m}$. Assuming that the frequency-domain channel remains unchanged on these $P$ resource elements, the frequency-domain signal collected by UE $k$ can be modeled as

$$
\left[\begin{array}{c}
y_{N_{1}} \\
\vdots \\
y_{N_{P}}
\end{array}\right]=\left[\begin{array}{ccc}
\Theta_{1, N_{1}} & \cdots & \Theta_{M, N_{1}} \\
\vdots & \ddots & \vdots \\
\Theta_{1, N_{P}} & \cdots & \Theta_{M, N_{P}}
\end{array}\right]\left[\begin{array}{c}
\beta_{1}^{1 / 2} \mathbf{g}_{1, N_{1}}^{T} \mathbf{w}_{1} \\
\vdots \\
\beta_{M}^{1 / 2} \mathbf{g}_{M, N_{p}}^{T} \mathbf{w}_{M}
\end{array}\right]+\left[\begin{array}{c}
z_{N_{1}} \\
\vdots \\
z_{N_{P}}
\end{array}\right]
$$

wherein

$$
\begin{aligned}
& \boldsymbol{\Omega}=\left[\begin{array}{ccc}
\Theta_{1, N_{1}} & \cdots & \Theta_{M, N_{1}} \\
\vdots & \ddots & \vdots \\
\Theta_{1, N_{P}} & \cdots & \Theta_{M, N_{P}}
\end{array}\right] \\
& \mathbf{f}=\left[\beta_{1}^{1 / 2} \mathbf{g}_{1, N_{1}}^{T} \mathbf{w}_{1}, \ldots, \beta_{M}^{1 / 2} \mathbf{g}_{M, N_{P}}^{T} \mathbf{w}_{M}\right]^{T}=\left[f_{1}, \ldots, f_{M}\right]^{T}
\end{aligned}
$$

Subsequently, using minimum mean square error (MMSE) channel estimation, the cross-correlation matrix of $\mathbf{y}_{n_{P}}$ and $\mathbf{f}$ is

$$
\mathbf{R}_{\mathbf{f y}_{N_{P}}}=\mathrm{E}\left[\mathbf{f y}_{N_{P}}^{H}\right]=\mathbf{C}_{\mathbf{f}} \boldsymbol{\Omega}^{H}
$$

and the autocorrelation matrix of $\mathbf{y}_{N_{P}}$ is

$$
\mathbf{R}_{\mathbf{y}_{N_{P}} \mathbf{y}_{N_{P}}}=\mathrm{E}\left[\mathbf{y}_{N_{P}} \mathbf{y}_{N_{P}}^{H}\right]=\boldsymbol{\Omega} \mathbf{C}_{\mathbf{f}} \boldsymbol{\Omega}^{H}+\frac{\sigma^{2}}{A} \mathbf{I}_{P}
$$

where $\mathbf{C}_{\mathbf{f}}=\operatorname{diag}\left(\sigma_{f_{1}}^{2}, \ldots, \sigma_{f_{M}}^{2}\right)=\operatorname{diag}\left(\beta_{1}, \ldots, \beta_{M}\right)$ is the covariance matrix of $\mathbf{f}, A$ is the pilot power.

Finally, the MMSE estimate of $\mathbf{f}$ is [18] 


$$
\hat{\mathbf{f}}=\mathbf{R}_{\mathbf{f}_{\mathbf{y}_{P}}} \mathbf{R}_{\mathbf{y}_{N_{P}} \mathbf{y}_{N_{P}}}^{-1} \mathbf{y}_{N_{P}}=\left(\frac{\psi}{\zeta} \mathbf{C}_{\mathbf{f}}^{-1}+\boldsymbol{\Omega}^{H} \Omega\right)^{-1} \boldsymbol{\Omega}^{H} \mathbf{y}_{N_{P}}
$$

where $\psi=E\left[\left|\mathbf{h}_{k, n}^{H} \mathbf{w}_{k}\right|^{2}\right] / A$ is the average signal-to-pilot power ratio, and $\zeta=E\left[\left|\mathbf{h}_{k, n}^{H} \mathbf{w}_{k}\right|^{2}\right] / \sigma^{2}$ is the average signal-to-noise ratio. Through DMRS interpolation, the MMSE estimate of UE $k$ can be obtained as

$$
\hat{\mathbf{y}}_{N_{\varphi}}=\boldsymbol{\Phi} \hat{\mathbf{f}}=\boldsymbol{\Phi}\left(\frac{\psi}{\zeta} \mathbf{C}_{\mathbf{f}}^{-1}+\boldsymbol{\Omega}^{H} \boldsymbol{\Omega}\right)^{-1} \boldsymbol{\Omega}^{H} \mathbf{y}_{N_{P}}
$$

wherein

$$
\boldsymbol{\Phi}=\left[\begin{array}{ccc}
\Theta_{1,1} & \cdots & \Theta_{M, 1} \\
\vdots & \ddots & \vdots \\
\Theta_{1, N_{\varphi}} & \cdots & \Theta_{M, N_{\varphi}}
\end{array}\right]
$$

Through calculation, the mean square error of channel estimation is

$$
\operatorname{MSE}_{\hat{\mathbf{y}}_{N_{\varphi}}}=\frac{1}{N_{\varphi}} \mathrm{E}\left[\left(\mathbf{y}_{N_{\varphi}}-\hat{\mathbf{y}}_{N_{\varphi}}\right)^{H}\left(\mathbf{y}_{N_{\varphi}}-\hat{\mathbf{y}}_{N_{\varphi}}\right)\right]=\frac{1}{N_{\varphi}} \operatorname{tr}\left(C_{\hat{\mathbf{f}}} \boldsymbol{\Phi}^{H} \boldsymbol{\Phi}\right)
$$

where $\mathbf{C}_{\hat{\mathbf{f}}}=\left(\mathbf{C}_{\hat{\mathbf{f}}}^{-1}+\frac{\zeta}{\psi} \boldsymbol{\Omega}^{H} \boldsymbol{\Omega}\right)^{-1}$ is the covariance matrix of the estimated channel $\hat{\mathbf{f}}$.

\section{Numerical results}

In this section, some numerical examples are evaluated to show the performance of the proposed channel estimation and precoding optimization under various system settings. We consider a cell-free massive MIMO-OFDM system with detailed simulation parameters listed in Table 1.

Figures 3 and 4 compare the achievable sum rate of the system when using traditional conjugate precoding and the proposed optimized precoding, where the precoding granularity is $2 R B, 4 R B, 6 R B$ and $8 R B$, respectively. In these two precoding schemes, the sum

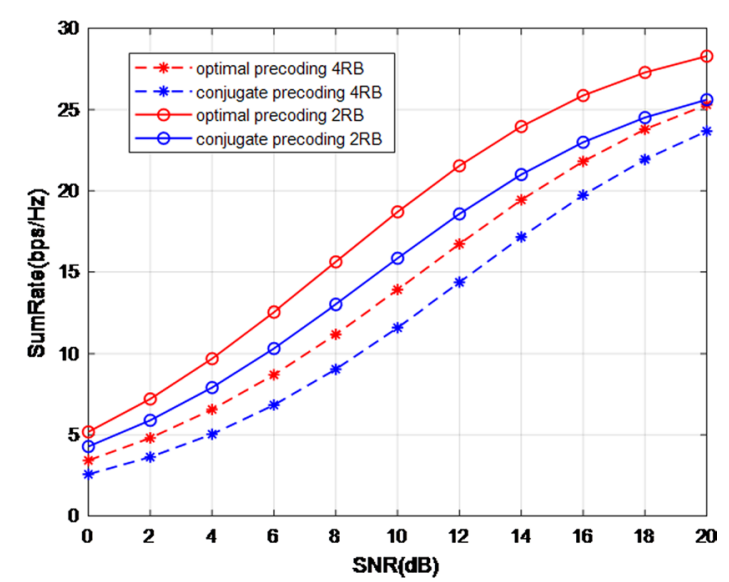

Fig. 3 Performance comparison of the multi-RB precoding 


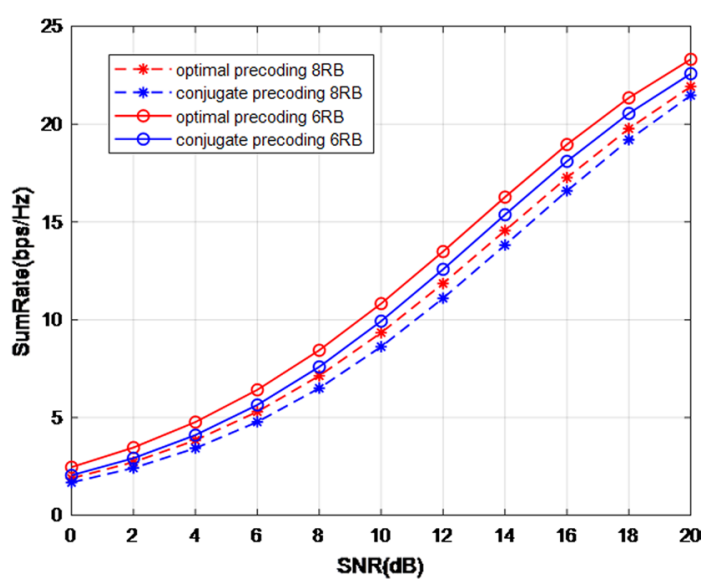

Fig. 4 Performance comparison of the multi-RB precoding

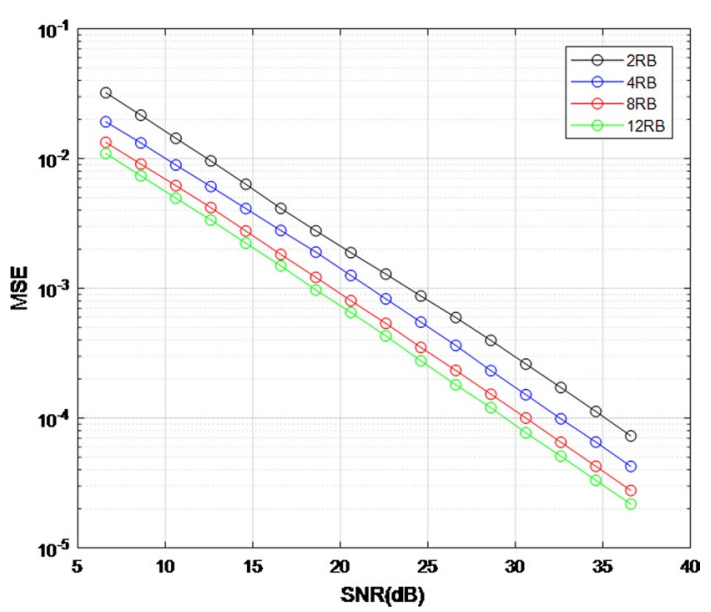

Fig. 5 MSE comparison of the multi-RB channel estimation

Table 1 Simulation parameters

\begin{tabular}{ll}
\hline Number of RAUs & 8 \\
Number of UEs & 4 \\
Number of antennas per RAU & 4 \\
Number of subcarriers per RB & 12 \\
Granularity of precoding & $2,4,6,8$ (RBs) \\
Granularity of channel estimation & $2,4,8,12,24$ (RBs) \\
Radium of background & $12(\mathrm{~m})$ \\
Path loss exponent & -3.7 \\
FFT points & 4096 \\
Power constraint for RAUs & $10(\mathrm{~W})$ \\
DMRS interpolation interval & 4
\end{tabular}

rate increases as the SNR increases and decreases with increasing granularity of the precoding. In addition, as expected, our proposed optimized precoding always performs better than conjugate precoding in terms of sum rate. When the precoding granularity is 
$2 \mathrm{RB}$ and $4 \mathrm{RB}$, the sum rate is increased by approximately 20 percent, and when the precoding granularity is $6 \mathrm{RB}$ and $8 \mathrm{RB}$, the sum rate is increased by approximately 10 percent. This result is because as the number of subcarriers increases, the impact of DSDs on system performance becomes increasingly serious. Since the minimum granularity of precoding in the existing $5 \mathrm{G}$ NR protocol is $2 \mathrm{RB}$ and is usually statically configured as $4 \mathrm{RB}$, the proposed algorithm can reflect the robustness to DSDs in wideband precoding under the existing $5 \mathrm{G}$ NR protocol.

Figure 5 shows the MSE vs. SNR performance comparison of the proposed downlink channel estimation when the channel estimation granularity is $2 \mathrm{RB}, 4 \mathrm{RB}, 8 \mathrm{RB}$ and $12 \mathrm{RB}$. It can be observed that as the SNR increases, the MSE of the proposed channel estimation decreases, and the accuracy of the channel estimation increases. In addition, the accuracy of the proposed channel estimation improves with increasing channel estimation granularity. This result is due to the use of DMRS interpolation intervals of the same width. The larger the bandwidth of the estimated channel is, the greater the number of DMRS participating in the channel estimation. Since the CSI-RS pilot can be designed in a single-port mode, the pilot overhead and design complexity are effectively reduced, thereby realizing low-complexity accurate estimation of the wideband channel with DSDs.

Figure 6 shows the performance comparison when the UE estimates the path loss and time delay based on the single-port CSI-RS sent by all RAUs, and different numbers of RAUs send DMRS. Assuming that the number of RAUs sending DMRS is 2, 4, 6 and 8 and the granularity of frequency-domain joint channel estimation is $2 R B, 12 R B$ and $24 R B$, it can be seen that the mismatch of CSI-RS and DMRS has little effect on the accuracy of channel estimation. Especially when the signal-to-noise ratio is low and the channel estimation granularity is small, the loss caused by this mismatch can be ignored.

\section{Conclusion}

In this paper, we have studied the DSDs problem in a cell-free massive MIMO-OFDM system due to its distributed characteristics, which causes the received signal to produce phase rotation related to the subcarrier index during demodulation. Since the existing

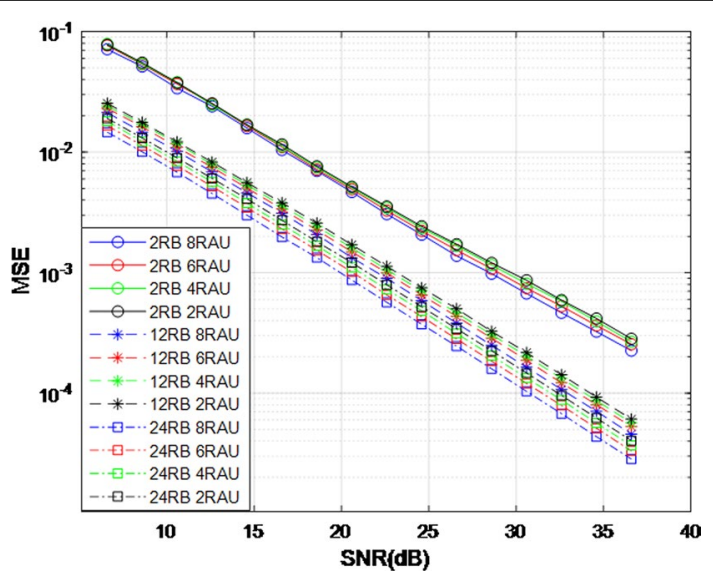

Fig. 6 MSE comparison of the multi-RB channel estimation 
5G NR protocol does not support per subcarrier precoding, to address the impact of DSDs, we designed a multi-RB precoding optimization problem and used convex approximation to solve the problem. Then, to cope with the influence of the DSDs on the accuracy of channel estimation, we propose a downlink channel estimation method, which uses CSI-RS and DMRS jointly to realize multi-RB channel estimation. The simulation results show that, compared with conjugate precoding, the proposed optimal precoding can effectively improve system performance. In addition, the proposed downlink channel estimation can obtain accurate channel information.

\section{Authors' contributions}

YG wrote the manuscript, simulated and modified the experiments; XX and DW helped to conceive the idea and revise the manuscript; PW and DL helped with article writing; ZF and AL contributed to the development of the ideas. All authors read and approved the final manuscript.

\section{Funding}

This work was supported in part by the Science and Technology Project of State Grid Corporation of China under Grant SGZJXT00JSJS2000454 and in part by the Support Plan for Postdoctoral Talents in Jiangsu Province and by the Project funded by China Postdoctoral Science Foundation under Grant No.2021 M702500.

\section{Availability of data and materials}

The datasets simulated and/or analyzed during the current study are available from the corresponding author on reasonable request.

\section{Declaration}

Ethics approval and consent to participate

Not applicable.

\section{Competing interests}

The authors declare that they have no competing interests.

\section{Author details}

${ }^{1}$ National Mobile Communications Research Laboratory, Southeast University, Nanjing 210096, People's Republic of China. ${ }^{2}$ State Grid Power Research Institute, Nanjing 211100, People's Republic of China. ${ }^{3}$ Ubiquitous Communication Research Center, Purple Mountain Laboratories, Nanjing 211111, People's Republic of China.

Received: 23 December 2021 Accepted: 6 February 2022

Published online: 02 March 2022

\section{References}

1. H.Q. Ngo, A. Ashikhmin, H. Yang, E.G. Larsson, T.L. Marzetta, Cell-free massive MIMO versus small cells. IEEE Trans. Wirel. Commun. 16(3), 1834-1850 (2017)

2. S. Buzzi, C. D'Andrea, Cell-free massive MIMO: user-centric approach. IEEE Wirel. Commun. Lett. 6(6), $706-709$ (2017)

3. E. Nayebi, A. Ashikhmin, T.L. Marzetta, H. Yang, B.D. Rao, Precoding and power optimization in cell-free massive MIMO systems. IEEE Trans. Wirel. Commun. 16(7), 4445-4459 (2017)

4. X. You et al., Towards $6 \mathrm{G}$ wireless communication networks: vision, enabling technologies, and new paradigm shifts, Sci. China Inf. Sci. 64(1), 74 (2021)

5. A.M. Hamza, J.W. Mark, E.A. Sourour, Interference analysis and mitigation for time-asynchronous OFDM CoMP systems. IEEE Trans. Wirel. Commun. 17(7), 4780-4791 (2018)

6. H. Yan, I. Lu, Asynchronous reception effects on distributed massive MIMO-OFDM system. IEEE Trans. Commun. 67(7), 4782-4794 (2019)

7. X. Liu, X. Zhang, Rate and energy efficiency improvements for 5G-based loT with simultaneous transfer. IEEE Internet Things J. 6(4), 5971-5980 (2019)

8. X. Liu, X. Zhang, NOMA-based resource allocation for cluster-based cognitive industrial internet of things. IEEE Trans. Ind. Inform. 16(8), 5379-5388 (2020)

9. X. Liu, X. Zhai, W. Lu, Celimuge Wu, QoS-guarantee resource allocation for multibeam satellite industrial internet of things with NOMA. IEEE Trans. Ind. Inform. 17(3), 2052-2061 (2021)

10. Y.S. Cho, J. Kim, W.Y. Yang, C.G. Kang, Synchronization for OFDM, in MIMO-OFDM Wireless Communications with MATLABR (IEEE, 2010), pp. 153-185

11. Y. Zhang, D. Wang, Y. Huo, X. Dong, X. You, Hybrid beamforming design for mmWave OFDM distributed antenna systems. Sci. China Inf. Sci. 63(9), 12 (2020)

12. W. Jiang, H.D. Schotten, Cell-free massive MIMO-OFDM transmission over frequency-selective fading channels. IEEE Commun. Lett. 25(8), 2718-2722 (2021)

13. L. Tran, M.F. Hanif, A. Tolli, M. Juntti, Fast converging algorithm for weighted sum rate maximization in multicell MISO downlink. IEEE Signal Process. Lett. 19(12), 872-875 (2012) 
14. M.S. Lobo, L. Vandenberghe, S. Boyd, H. Lebret, Applications of second-order cone programming. Linear Algebra Appl. 284(1-3), 193-228 (1998)

15. S. Boyd, L. Vandenberghe, Convex Optimization (Cambridge University Press, Cambridge, 2004)

16. 3GPP. Radio Physical Layer Aspects. Release 15, TR 21.915 V15.0.0 (2019)

17. Baoguo Yang, K.B. Letaief, R.S. Cheng, Zhigang Cao, Channel estimation for OFDM transmission in multipath fading channels based on parametric channel modeling. IEEE Trans. Commun. 49(3), 467-479 (2001)

18. S.M. Kay, Fundamentals of Statistical Signal Processing: Estimation Theory (Prentice-Hall, Englewood Cliffs, 1993)

\section{Publisher's Note}

Springer Nature remains neutral with regard to jurisdictional claims in published maps and institutional affiliations.

Submit your manuscript to a SpringerOpen ${ }^{\circ}$ journal and benefit from:

- Convenient online submission

Rigorous peer review

- Open access: articles freely available online

- High visibility within the field

Retaining the copyright to your article

Submit your next manuscript at $\boldsymbol{\Delta}$ springeropen.com 